\title{
Integration for Heterogeneous Manufacturing Information Systems Based on Semantic Entity
}

\author{
Song Yu-chuan ${ }^{1}$, Xiong Jun ${ }^{1}$, LI Xian-wang ${ }^{2}$ and Chen Xue-hai ${ }^{3}$ \\ ${ }^{1}$ State Key Laboratory of Mechanical Transmission, Chongqing University, \\ Chongqing, China, College of Mechanical Engineering \\ ${ }^{2}$ Guangxi University, Nanning, Guangxi, China \\ ${ }^{3}$ Research and design institute of agricultural machinery engineering, Hubei \\ University of Technology, Wuhan, Hubei, China \\ syc@cqu.edu.cn,741288920@qq.com,xianwang@gxu.edu.cn,cxh@cqu.edu.cn
}

\begin{abstract}
To support semantic integration for heterogeneous information systems in manufacturing enterprises, based on semantic gateway, the doncept of semanic entity is defined, and the semantic entity-based integration method is stydied. Semantic entity sets up conceptual structure by aggregating semantic concept from semantic gateway, and builds dynamic conceptual structure through adding or removing its general concept. By means of key business data, semantic entity identifies business object and obtains business data from its application system, and then instantiates concept structure. Based on instantiation, semantic service can obtain parameters dato from business object instant, and satisfies the interface contract. Semontic entity captures data changes of business object, and then establishes status-based integration rules, defines algorithm logic of rules. By instantiating concept structure aimed to specific application system and invoking semantic service, the integration ctivities among information systems come true. This work has been verified by prototypesystem, and attained expected integration effect.
\end{abstract}

Keywords: semantios; integration, gateways; mapping; framework

\section{Introduction}

With the continuous deepening of manufacturing information, the role of information technologies in the basiness activities of manufacturing enterprises is becoming more and more important. On the one hand, the various business aspects of manufacturing enterprises rely more and more on information systems. Internal activities from designing, process planning, and manufacturing to managing are all carried out with the support of corresponding information systems. On the other hand, due to the continuous development of information technologies, there exist so many information systems in enterprises. However, because of the phased implementation of manufacturing information and the lack of a unified industry standard for the development and implementation of manufacturing information system, most of these systems were developed and implemented by different service providers based on their own understanding of the business. Therefore, they are always heterogeneous in most cases, reflected on the one hand in the structure of the business objects, on the other hand in the semantics of business objects. In order to play the overall effectiveness of information systems, it is urgent for enterprises to realize their integration of the information systems[1]. Meanwhile, faced with a complex and volatile market environment, enterprises must be able to adjust their business activities to meet the needs of the market at any time and it will be very difficult to accomplish these business activities without the support of information systems. Therefore, this puts forward higher request to the ability 
of integration and collaboration of systems. Traditional integration approaches can hardly meet the business needs of modern manufacturing enterprises, and semantic-based integration has become the main development direction[2,3].To solve the integration issues manufacturing enterprises are facing, academics have done a lot of research. Literature [4] proposed an integrated approach of intelligent objects; Literature[5] proposed a method for business process integration; Literature[6] studied the integration approach between model-driven and service-oriented architecture. Meanwhile, semanticbased integration, service-oriented architecture and cloud manufacturing have been hot research fields. Literature[7] studied the peer semantic gateway integration method; Literature[8] studied the semantic integration method based on ontology mapping; Literature[9] conducted semantic extension to service-oriented architecture; Literature $[10,11]$ studied the applications of service architecture-oriented integration; Literature $[12,13]$ discussed the integration technology based on cloud computing and the future trends; Literature[14] studied the service shared integration model under cloud manufacturing cases. The above studies focused more on integration architectures, as well as on the expansion based on these architectures. Effective methods to solve the problem of semantic heterogeneity between different systems are still lacking, Aiming at the existing integration problem, the research group involving the autho proposed the concept of semantic gateway and studied its enabling and application tecbuologies. Then under the support of semantic gateway, semantic entity is put forward and built, providing a new way for the integration of information systems.

\section{Integrated basis and Framework of Manoacturing Enterprise Heterogeneous Information Systems based on Semantic Entity}

Semantic gateway is a kind of semantic thtegration platform for heterogeneous information systems, through the concepts and data mapping of information systems to realize semantic equivalence exchange of information, through the unified package and exposure to information systems services to realize consistent service access and stable contract. The concept semantic method of semantic gateway is shown in Figure 1, with Ai for the concept of system $\mathrm{A}, \mathrm{Bj}$ for the concept of system B, Gk for the concept standards of semantic gateway $\mathrm{G}$

In order to establish semantic links between the concept $\mathrm{Ai}$ and $\mathrm{Bj}$ of different systems, they must bain concept mapping relationship with the concept standards Gk separately, then through the concept landards to realize equivalence semantic exchange between different system concepts.

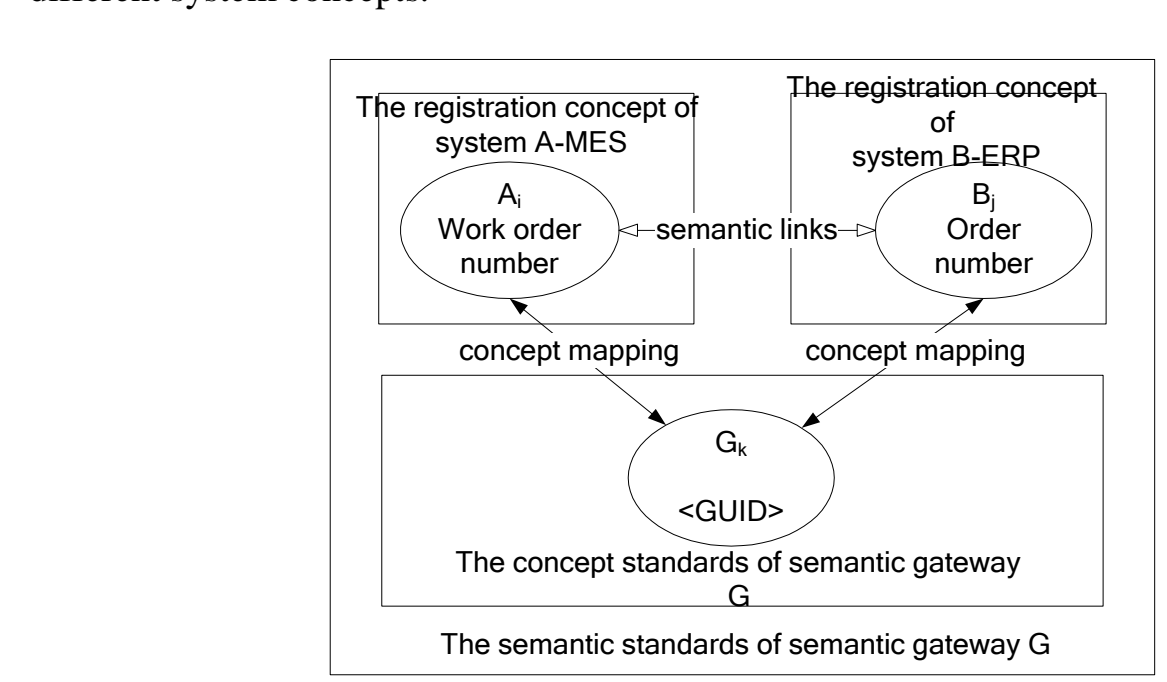

Figure 1. The Concept Semantic Method of Semantic Gateway 
There are two kinds of mapping relationship between the semantic gateway and the application system concept: When the calculating mapping function exists between the concepts, semantic equivalence relation can be calculated by the semantic gateway automatically through the mapping function; otherwise, the equivalence relation must be specified in the associated data of the concept.

To realize the semantic mapping and analysis between the systems, the enterprise information systems must be registered semantic concepts and semantic data in semantic gateway, meanwhile provide the exposed service interfaces and invocation path. So there exists the registration semantic information and service of information systems in semantic gateway.

Semantic gateway is a foundation platform which is independent of specific application systems; but in their source systems, there are a certain business semantic relations between most of the registration semantic information and service in semantic gateway. The relations were interrupted in semantic gateway. So it is obviously beneficial to semantic processing to reconstruct the semantic relations in semantic gateway. Because of the independence of the semantic gateway and its various semantic sources from heterogeneous systems, the semantic relations although derived from the specific systems, but couldn't depend on them. So the concept of semantic entity is put lorward to reconstruct the semantic relations better for semantic processing

\subsection{The Definition of Semantic Entity}

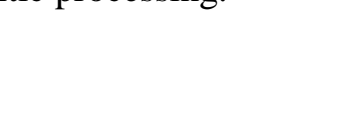

Definition: The semantic entity $\mathrm{SE}$ is a tetrad: $\mathrm{SE}=(\mathrm{C}, \mathrm{S}, \mathrm{R}, \mathrm{A}) . \mathrm{C}$ is the semantic concept, $\mathrm{S}$ is the semantic service, $\mathrm{R}$ represents the semantic integration rule, A represents semantic integration activities.

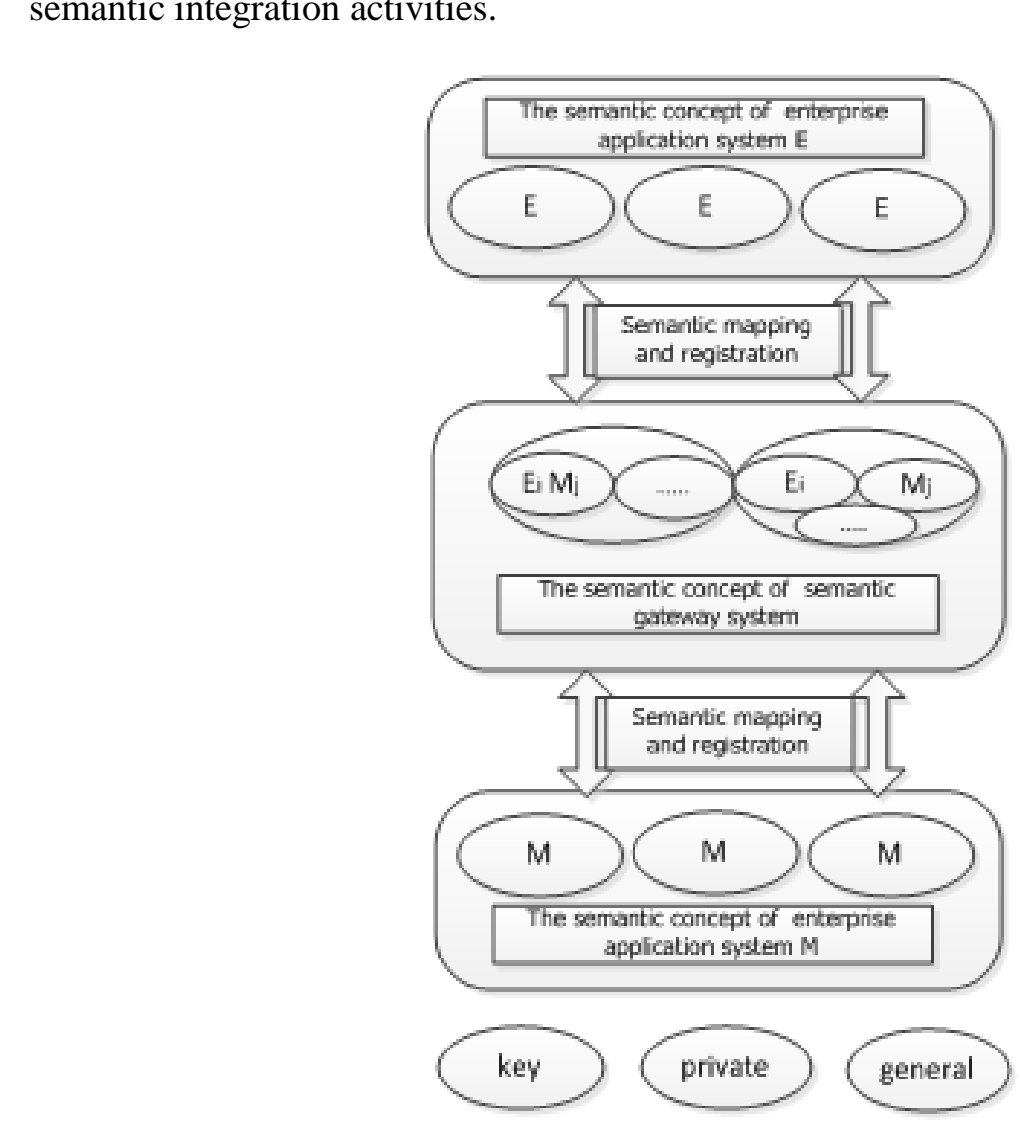

Figure 2. The Semantic Concept Registered Relationship between Application System and Semantic Gateway 
Because of the semantic entity was established based on the semantic gateway, its semantic concept $\mathrm{C}$ derived from the semantic concept which the information system registered in semantic gateway. The relations between the business objects involved in semantic integration of enterprise information systems and the semantic concept registered relationship among the semantic gateways is shown in Figure 2.And the semantic concept of business objects can be divided into three categories, respectively the key concepts, general concepts, and private concepts. The key concepts are the concepts which can uniquely identify the business objects. They must be registered in the semantic gateway, and the semantic gateway establishes semantic relations through the key concepts and specific business of the application systems. The general concepts are the non-critical business concepts which are registered in the semantic gateway. They tend to describe the specific status of business objects. But private concepts refer to the business concepts which have not been registered in the semantic gateway, and they can be only understood by their own application systems.

Based on the classification of the concept of business objects of the application system registered in gateway. The concept of semantic gateway divided into two categories, key concepts and general concepts, which contain the concept registered by all the all application system business objects. There are semantic relations between the key concepts of different application systems with a business integration denfand, and there is the semantic equivalence relationship between most general concepts

Semantic service $S$ is the functional service which associated with the business object of current semantic entity. This service can extend the semantigentity ability, and perform specific business logic based on semantic data information. The semantic service of the semantic entity come from the service which application system registered in semantic gateway. This service has the stable interface contract and semantic after encapsulation by the semantic gateway.

Semantic integration rules $\mathrm{R}$ refers to assefies of business logic comparison conditions. It can get true or false logic value through lesting the state before and after of semantic data changes. Then it cargecide whether or not trigger subsequent integration activities or execute service semantics. An integration rule can trigger multiple integration activities or semantic services at the same time

A semantic infegration activity $A$ is the specific practitioner of the semantic integration between systems by the semantic entity. With the support of semantic gateway, they build the business object which donform the target system semantic participating in the integration according to the corresponding business object of semantic entity, and distribute and synchronize between different information systems. The integration activities execute automatically after triggered by integration rules.

\subsection{Heterogeneous Information Systems Integration Framework based on Semantic Entity}

The proposed semantic entity provides a new way for the use of semantic knowledge in semantic gateway, in order to give a better support to the integration of heterogeneous information systems, a heterogeneous information systems integration framework of manufacturing enterprise based on semantic entity is proposed and described as Figure 3. 


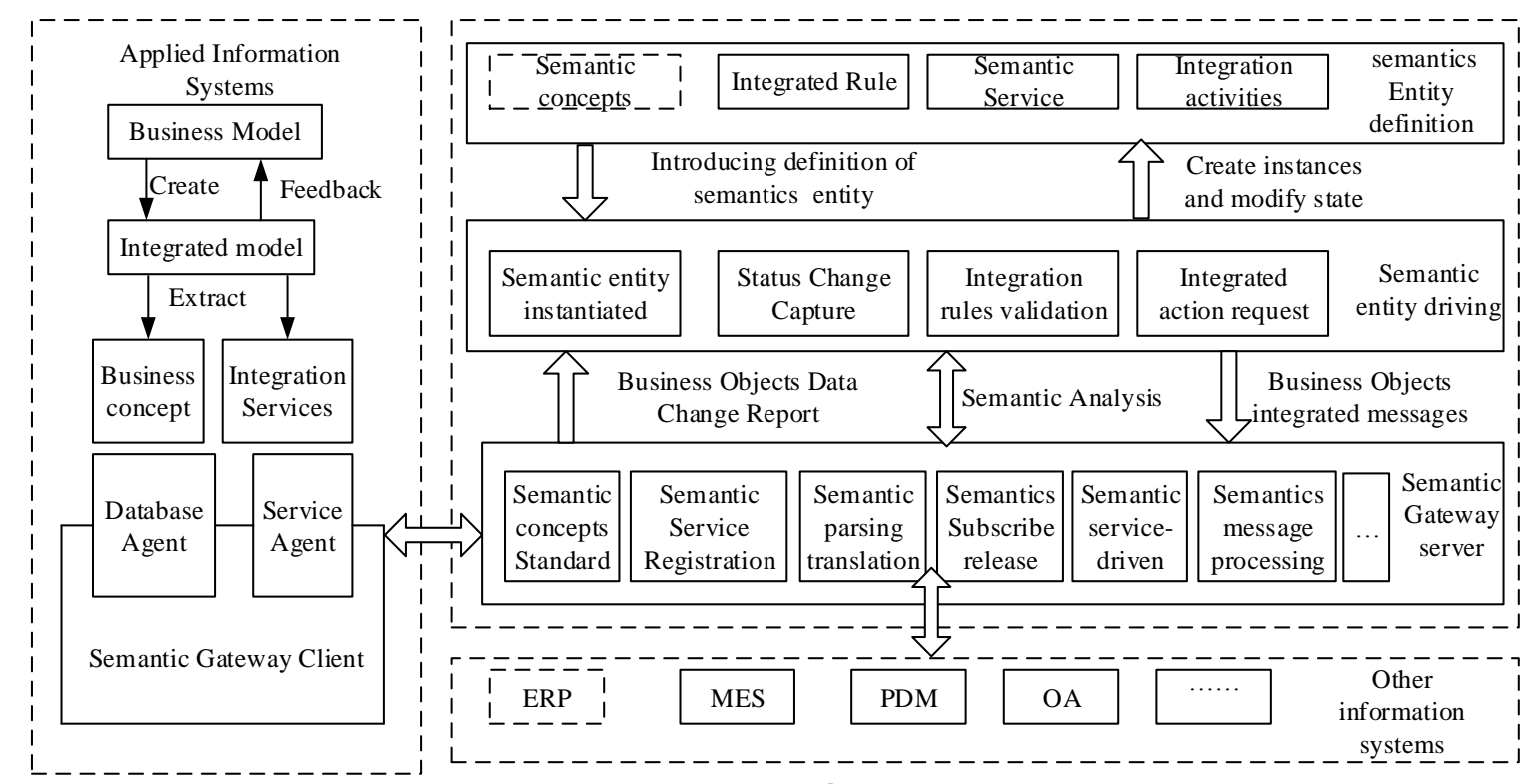

\section{Figure 3. Heterogeneous Information Systems integration Framework based on Semantic Entity}

The heterogeneous information systems integration framework of manufacturing enterprise based on semantic entity is composed of enterprise application information systems, semantic Gateway Client, Semantic Gateway Server, semantic definitions and semantic entity drive and other component

Enterprise application information systems refer to 2 yariety of systems which support their business running, such as ERP, MES and PDM. By analyzing the business logic of the application of information sysems, an enterprise business model on the application of information systems can be obtained. Besides, the analysis above can help us decide which business objects and which concepts and functions of these business objects are involved in the integration between systems. These two parts results in an integrated model of business.

Semantic Gateway Client and enterprise applications work together by monitoring, accessing and apdating the business objects of the application system, invoking integration seryices the application systems exposed, and exchanging semantic information with semantic Gateway Server.

Semantic Gateway Server is used to achieve semantic processing of a variety of semantic integration messages. The establishment of standards and concepts of semantic mapping between conceptual in Semantic Gateway Server make semantic parsing and conversion achievable, meanwhile, the server can package integration services provided by application system and provide a unified semantic stable access interface. Then, simultaneous distribution of semantic message can be realized based on the semantic subs ription of information systems. When semantic Gateway Server receives state changes of a business object, it not only provides the message to the driver module of semantic entity, but also receives the integrated messaging of this business object from semantic entity drive module, thus it can act as an agency which provides the integrated service of semantic entity .

The definition of semantic entity, which is responsible for controlling the abundance of semantic concepts of semantic entity so as to meet the demand of different system integration based on integrated activities of certain semantic structure.is used to maintain the structure of the semantics.

Semantic entity driver realized the semantic integration operation, through the concept of semantic entity data instantiation, get the state of the specific business object information, and based on the business object state changes before and after the test of all 
the principles of integration, and perform the eligible integration activities and semantic services.

With the support of integration framework, semantic entity can make full use of the advantages of the semantic gateway semantic integration, realize the loosely-coupled integration based on semantics between application systems, the specific method to realize the integration will be discussed in subsequent sections.

\section{Semantic-Entity-based Manufacturing Enterprise Information System Integration of Heterogeneous of Implementation}

\subsection{Conceptual Structure Dynamically build of Semantic Entity and Implementation of Examples}

Since the concept of semantic entity comes from the standard of semantic concepts of semantic gateway registration, and the concepts of semantic gateway divided into two categories of key concepts and general concepts, the concepts of the semantic entity can be divided into two categories. The key concept of the semantic entity is used to identify the specific business objects, which are shared by different applications. You can establish the semantic integration contact related business objects in different application systems through key concepts of semantic entity key concept is the core concept of structure of the concept of the semantic entity. The general concept of semantic entity is used to describe the status information of the semantic entity, those come from the general concept of the semantic gateway.

The dynamic construction of the concept structure of semantic entity refers to the general concept of the structure defines of the semantic entity concept of a dynamic combination involved the key concepts, so that the semantic entity produces the structure containing various general concepts, meeting the needs of different systems integration. In the production order, for exanple, in Figure 4 shows the specific construction method. The "order number" is the key concept "produce, quantity, completed number, verification status" are functioned as general concepts, among these "Order Number" must be included in the concepror the dynamic semantic structure of each entity, the rest general concepts can be further free combination, which can generate different conceptual structure.

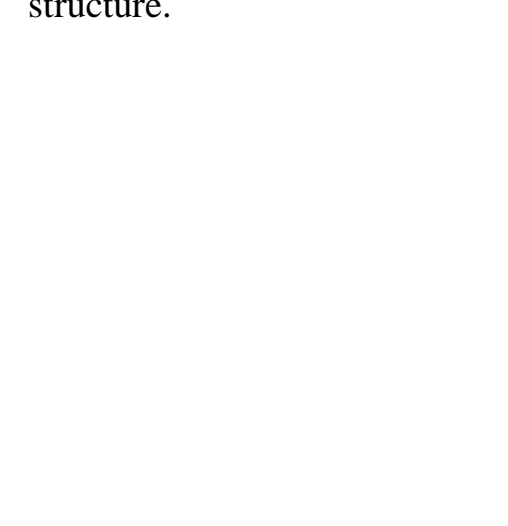




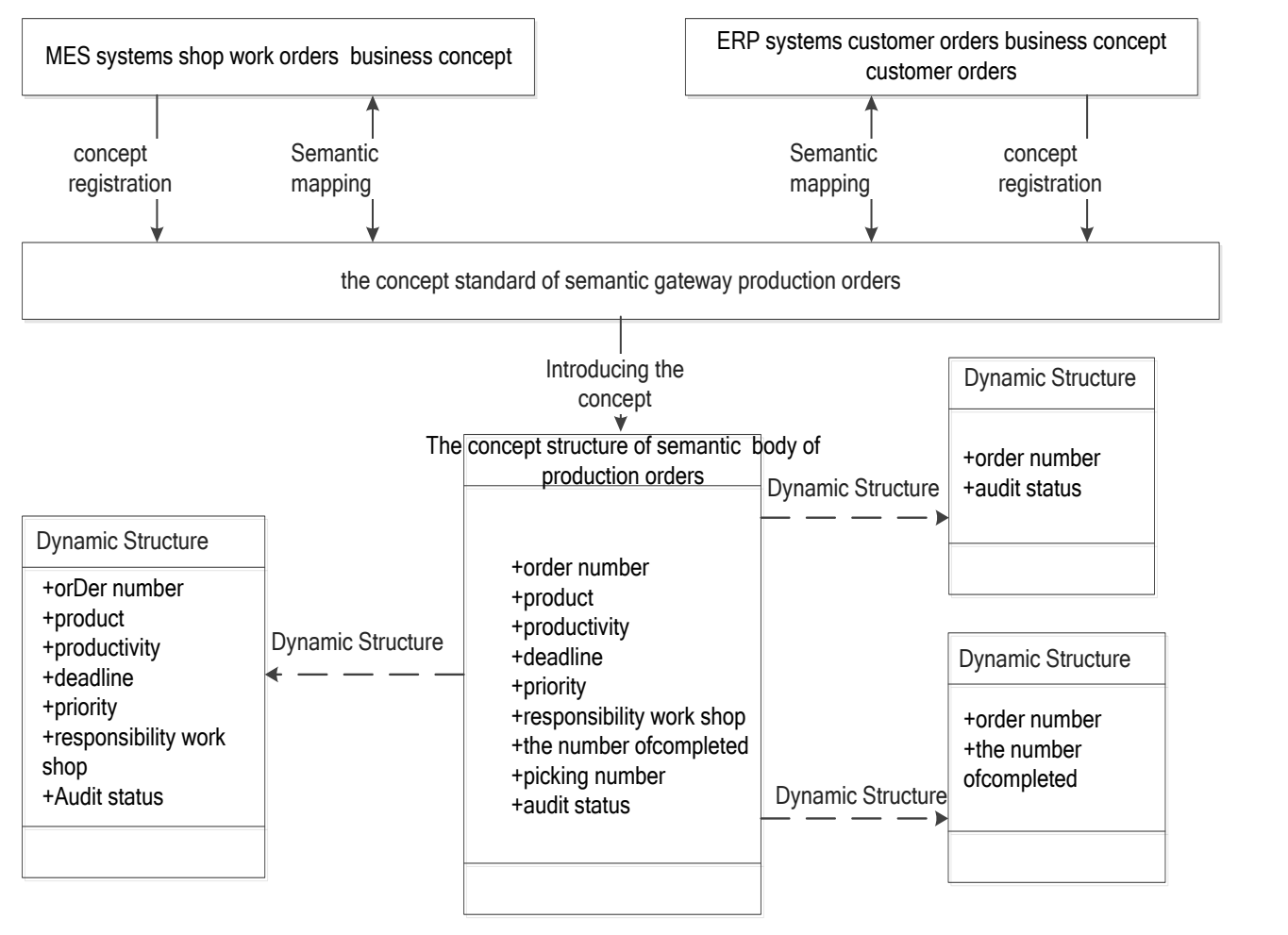

Figure 4. Conceptual Structures Dynamically Build of Semantic Entity

Conceptual structure defines the semantics of the entity among the base prototype system business integration requirements, examples of semantic entity is based on the concept of access to key concepts and concretesstructure is defined by the semantics of the data from the application gateway correct information and business data to populate the semantic entity, shown in Figure 5.

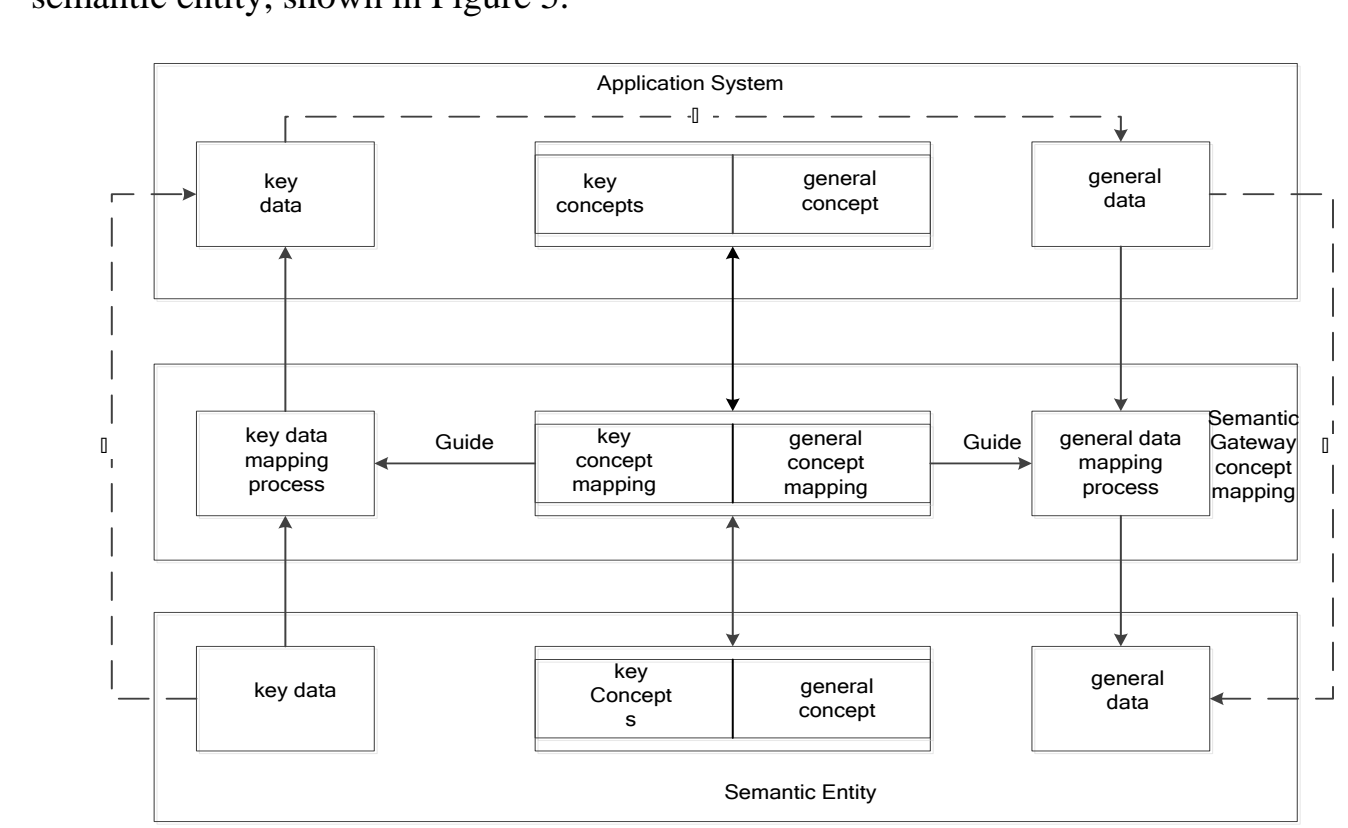

Figure 5. Examples of Structure Concept of the Semantic Entity

First, the semantic entity must include the key semantic concepts of critical data through the key concepts of semantic mapping schemes between the concept and application of the concept of critical data semantic entity mapping process critical data for 
the target application system, which can be able to determine the specific business objects of the application system. Then, based on the general concept of the semantic structure of the concept of dynamic semantics contained in the entity, we can identify them separately from the main application system. Finally, based on the general concept of mapping scheme among the data from the application of the general system and converted them to the general entity of data semantics, and filled them into a dynamic concept semantic structure of the entity.

\subsection{The Semantic Entity Implementation Method of Semantic Service}

Semantic entity's semantic services come from the service which different information system registered in semantic gateway, these services have the strictly contract to access, because of the semantic entity was built on the basis of business integration demand, so the introduced concept of service and semantic structure have a close business relationship. There exists semantic correspondence between the service's interface parameters and the concept of semantic entity, and instantiated data from semantic entity can be used as input parameters of the service to invoke these services. With the support of semantic gateway, semantic services processing of semantic entity is shown in Figure 6.

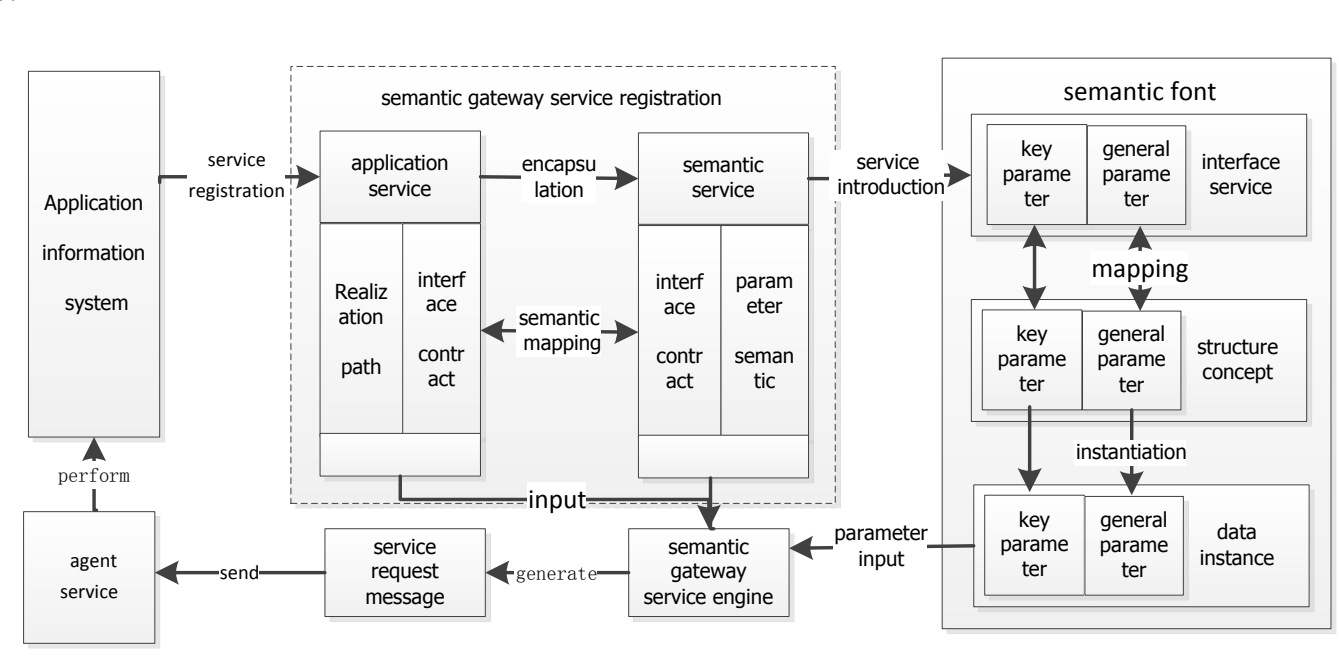

Figure 6. The Semant Entity Implementation Method of Semantic Service

The interface contract and realizing route which Application Information System register service in semantic gateway: Realizing route defines invoke address of service, and interface contracts define service invocation semantics. Semantic gateway semantic mapping sefvice interface contracts parameters of the application system, make the semanticparameters by application systems can be converted into semantic gateway, and then encapsulate application service interface contracts, to provide a service access interfaces unrelated with the semantic of application system.

Because of the correspondence between the semantic entity's service parameters and concept, when a service execution request appeared, semantic entity automatically obtain input parameters from the corresponding conceptual data instance interface contract services based on semantic gateway exposed. When the input parameter information can not satisfy the interface contract, semantic entity parameter data can be automatically acquired from the application of the system according to the needs of the instantiation principle, so that the service parameter information is complete. After semantic gateway service engine obtaining the semantic service interfaces and complete parameter input data, then restore the data from the service semantics of semantic gateway to the service semantics of Application Information System according to service pack relations and add 
the request message generated by invoke address of service. Finally, the semantic gateway to send a message to the service agent deployed on the target application system, executed the actual service by service agent, thus completing the service execution of semantic entity.

\subsection{Establishment and Trigger Implementation of Semantic Entity's Integrated Rule}

Semantic integration rules based on the state of the business object changes, the integration rule is the definition of the state of the business object changes. When the application system of business data changes, the data changes will be caught by the client agent of semantic gateway, then the information delivered to semantic gateway server, and then send them to the semantic entity after semantic gateway semantic processing the information, semantic entity instantiate the dynamic structure of the business object according to change of business information, and input them into the rule logic processing module of semantic entity at the same time. Establishment and trigger implementation of Semantic entity's integrated rule as shown in Figure 7.

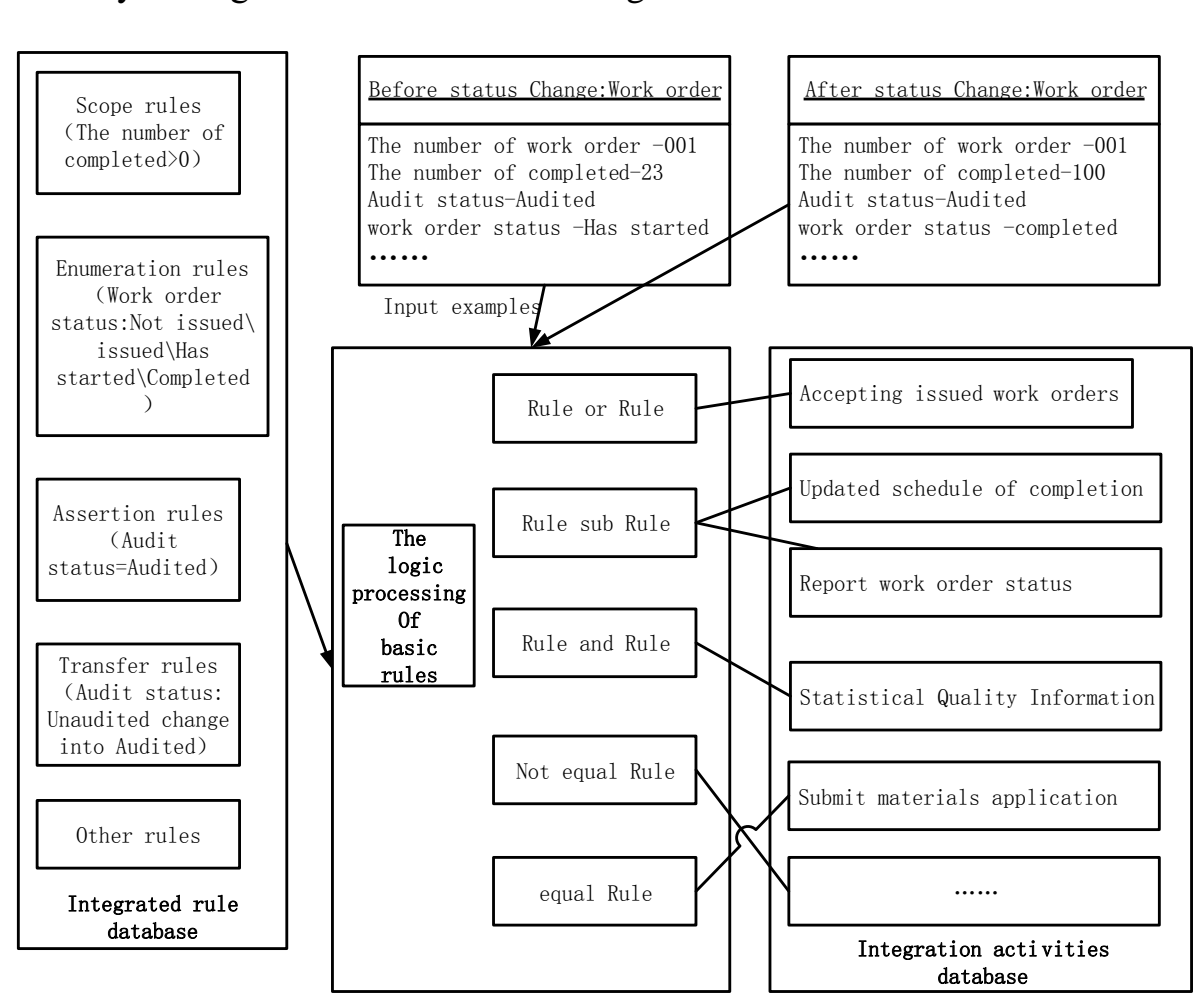

Figure 7, Establishment and Trigger Implementation of Semantic Entity's Integrated Rule

Ineg ation rules of semantic entity are divided into four categories respectively is scope rules, enumeration rules, assertion rules and transfer rules, etc. Scope rules defines the attribute value interval of trigger, used to describe the continuous changes in the properties of the state; Enumeration rules defines the attributes of the limited collection value, is used to describe attribute characteristic of fixed value; Assertions rules equivalent to determine the attribute value, is used to describe various key properties; Transfer rules defines attribute value change of state, describes the attributes from one state to another state.

Through the establishment of the integrated rules, business entity rule logic processing can be carried out based on the business object instance state. The basic logic processing is divided into "equal" and "not equal" operation by single rule, "or", "and" , "sub" operation by two rules and complex logic operations based on multi-rules. "equal" means 
business state satisfy integration rules; "not equal" means business state not satisfy integration rules; "or" means business state meet one of two integration rules; "and" means business state satisfy the two integration rules at the same time; "sub" means business state meet the preceding rule but not meet the following rule at the same time. Each integration regular expressions can be associated with one or more integration activities. The results of logical processing decide whether to implement integration activities associated with integration rules. When the calculated value of integration rules is "true", the business entity will automatically submit request of integration activities.

By constructing semantic entity dynamic concept structure instantiation, semantic entity can get state data of instance status changes before and after from the semantic gateway, and according to the current semantic entity's all integration rules, semantic entity test the state of instance changes and get all the conditions which meet the state's integration rules. Then according to the integration rules corresponding to integration activities to execute business object integration between systems.

\subsection{Method of Integration Activities Creation and Drive Implementation of S mantic Entity}

Semantic entity integrated rules define conditions and needs of busines integration and semantic entity integrated activities provide foundations to realize business integration. Semantic entity integration activities are divided into tyo parts. One part is to operate the business object instance of semantic entity based on the concept structure of the semantic entity, and another part is to visit the semantieservice defined by semantic entity. They are both based on instance construction operation of semanticentity, as shown in Figure 8.

Instance construction operation is composed of quelying, creating, and modifying; querying operation is used for extracting attribute values from the application

system according to key dataceating operation is used for creating and filling semantics entity instances; modifying operation is employed for changing the state of the instances and reporting the instances staes before and after the changes to the semantic gateway.

Instance integrated operations of semantic entity consist of creating, updating, and deleting operations, Creating eperation is used to establish new instance data of business objects in the target integrated system; updating operation is responsible for submitting instance data to the target system and updating its business status data; deleting operation removes invalid business objects from the integrated system.

Semantic service of semantic entity can achieve heterogeneous service integration among systems. Integrated services of semantic entity depend generally on the instances of the semantic entity, and semantic entity is able to build business instance by critical data. So semantic service based on semantic entity can automatically obtain its necessary executive parameters to satisfy the parameter constraints on service calls, and the service also support parameter semantic call requests. 


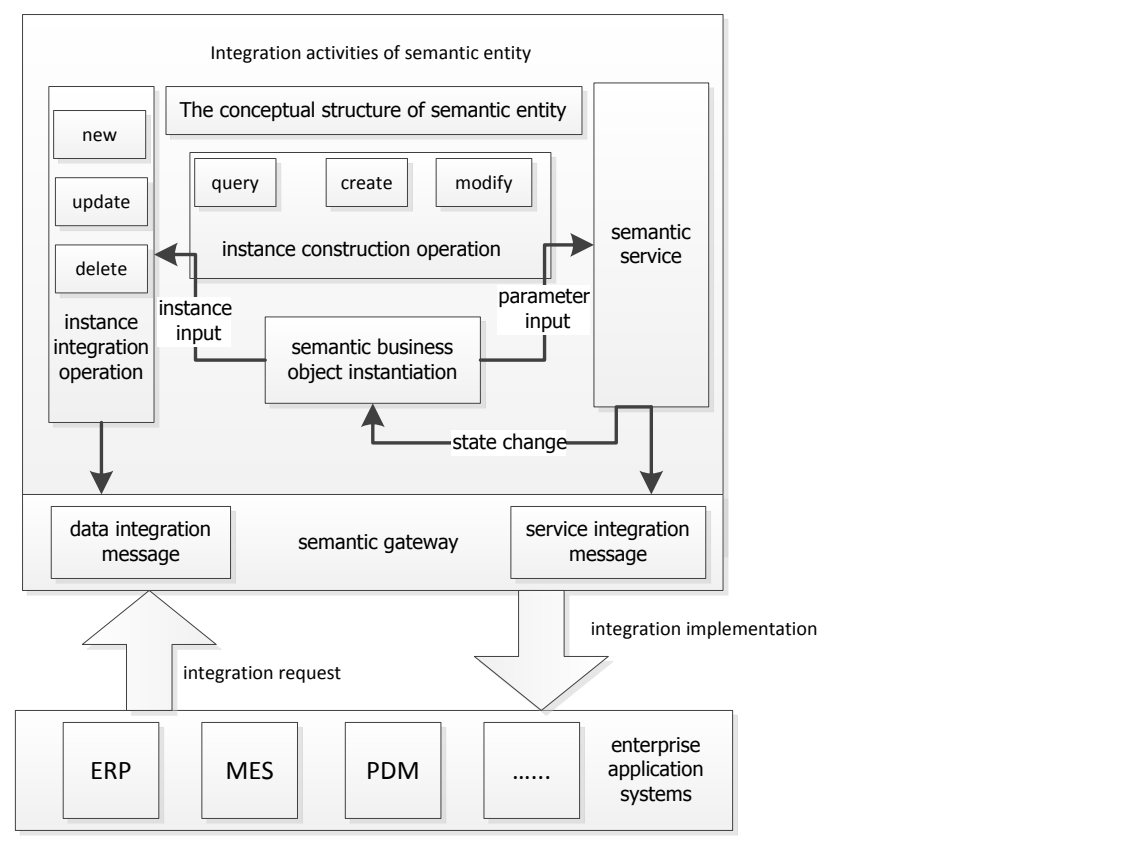

Figure 8. Semantic Integration Activities Ceation and Drive Implementation

When the semantic entity receives the integrated request the integrated activities will be firstly classified. If it is data integration between businesses objects, the semantic entity will create business instances automatically based on the definition of integration rules, submit the instances to semantic gatevay for semanticp pocessing and then send them to the target systems; If it is the services integration the semantic entity will automatically analyze semantics of service requests. When the semantic information is not enough, the semantic entity will obtain the missing datafrom the application system automatically based on the critical data of request information to support service calls.

\section{The Application Cases}

Based on the study on the integration framework and implementation technologies of semantic entity the author has developed prototype system of the semantic entity integration and application verification has been conducted. Heterogeneous information systems integration in manufacturing enterprises based on semantic entity is a kind of integration with loose coupling and flexibility. Only by providing semantic information to the semantic gateway and registering semantic service, the application system can complete the preparatory work to participate in the integration with full compatibility, without modifying the legacy system code, thereby maintaining the system's stability and independence. Supported by semantic entity enabling tools, semantic integration calls from information systems can be achieved by defining the conceptual structures and semantic services, integration rules and integration activities.

Taking the automatic issue of work orders from ERP to MES in a manufacturing enterprise for example, the integration process based on semantic entity is as follows.

Firstly, ERP and MES will both respectively register key concepts of work orders like "work order number" and " serial number" and general concepts like "product number, expected production number, expected start time" and "part number, tasks number, planning start time" with semantic gateway, and will establish semantic mapping relationship with semantic concepts of work orders in the semantic gateway like " product, process number, planning start time", thus making the semantic gateway understand their semantics and realizing mutual resolved. 
Secondly, with the support of integration enabling tools of semantic entity, semantic concepts involved in issue integration will be got from semantic gateway based on analyzing the integration requirements of work orders, then semantic entity based on work orders business will be constructed, which includes semantic concepts like "worksheet number, product, quantity, responsibility workshop, process planning, completed number, planning start time, verification status".

Then State transition integration rules of "verification status" will be built: " verification status: unverified to verified" and the integration activities "create and fill the semantic entity instance of work orders for MES" will also be constructed. The integration activities will create a new work order in MES, create worksheet number as rules automatically, establish semantic relationship with work order number in ERP by conceptual standards of semantic gateway, and as well fill the data information of general concepts, thus completing the creation of work orders instance.

Finally Trigger relationship between the above integration rules and integration activities will be built, and when the status changes of work orders semantic entity meet the transfer rules of "verification status", integration activities will be implemented.

When work orders from ERP get through an initial audit, their " verification status" will shift to meet the integration rules and automatically trigger the in egration activities and work order business instance will be submitted to MES, by adding work order to MES through semantic gateway, finally achieving issae integration of wotk order.

\section{Conclusions}

Semantic integration is the developmentrend of heterogeneous information systems integration in manufacturing enterprises. Semantic gateyay provides a semantic mapping and analytic platform for the semantic integration between the information system, as well as a unified integration interface contracts, and the realization method for the integration of systems. With the support of senantic gateway, Semantic entity provides an integration application method of semantic concepts and semantic service. Through organizing the semantic concepts and semantic service and defining the integration rules and integration activities, Semantic entity can provide a dynamic, flexible and loosely-coupled integration way based on the state of business objects. By establishing different dynamic conceptual structure and integrate activities for different application systems, semantic entity can meet the needs of semantic integration needs among many different systems at the same time, thereby reducing the eost and complexity of the realization of the integration, and improving the maintairabjlity of the integration relationship, and the effectiveness has been verified through the development and implementation of the prototype system.

\section{Acknowledginent}

The research is supported by the National Natural Science Foundation of China (NSFC) under Grant No. 51205429 and the Technology Pillar Program during the 12th Five year Plan Period of China under Grant No. 2012BAF01B01 .

\section{References}

[1] Hvolby H, Trienekens J. Challenges in business systems integration[J]. Computers in Industry, 2010, 61(9): 808-812.

[2] Panetto H, Molina A. Enterprise integration and interoperability in manufacturing systems: Trends and issues[J]. Computers in Industry, 2008, 59(7): 641-646.

[3] Chen David, Doumeingts G, Vernadat F. Architectures for enterprise integration and interoperability Past, present and future[J]. Computers in Industry, 2008, 59(7): 647-659.

[4] Zang Chuan-Zhen, Fan Yu-Shun. Information system of manufacturing enterprise based on smart items[J]. Computer Integrated Manufacturing Systems, 2007, 13(1):49-56.

[5] Li Qing, Zhou Jian, Peng Qi-Rui, et al. Business processes oriented heterogeneous systems integration platform for networked enterprises[J]. Computers in Industry, 2010, 61(2): 127-144. 
[6] Ricardo J, Antonoi G, Adolfo S. Challenging the interoperability between computers in industry with MDA and SOA[J]. Computers in Industry, 2006, 57(8-9): 679-689.

[7] Zhou Jing-Tao, Yang Hai-Cheng, Wang Ming-Wei, et al. Peer to peer semantic grid service oriented enterprise information integration architecture[J]. Computer Integrated Manufacturing Systems, 2010, 16(12): 2697-2707.

[8] Zhao Zhen, Yan Jun-Wei, Liu Min, et al. Enterprise semantic integration \& business collaboration platform oriented to steel continuous casting equipment $\mathrm{MRO}[\mathrm{J}]$. Computer Integrated Manufacturing Systems, 2010, 16(10): 2044-2056.

[9] Yu Peng-Fei, Song Xiao, Zhang Lin, et al. Architecture for information integration system based on semantic SOA[J]. Computer Integrated Manufacturing Systems, 2009, 15(5): 959-967.

[10] Jiang Yan-Chuan, Gong Xiao-Rong, Yin Chao. Integration framework and implementation technologies of integrated workshop production management system for motorcycle parts enterprise[J]. Journal of Chongqing University, 2011, 34(8): 71-79.

[11] Kuk Seung-Hak, Kim Hyeon-Soo, Lee Jai-Kyung, et al. An e-Engineering framework based on serviceoriented architecture and agent technologies[J]. Computers in Industry, 2008, 59(9): 923-935.

[12] Marstom S, Li Zhi, Bandyopadhyay S, et al. Cloud computing — The business perspective[J]. Decision Support Systems, 2011, 51(1): 176-189.

[13] Xu Xun. From cloud computing to cloud manufacturing[J]. Robotics and Computer-Integrated Manufacturing, 2012, 28(1): 75-86.

[14] Guo Gang, Yang Juan, Zhang Yi, et al. Design and analysis of the manufacturing resource services sharing model based on the service-oriented manufacturing paradigm[J]. Journal of Chongqing University, 2012, 35(9): 48-55.

[15] Song Yu-Chuan, Lei Qi, Liu Fei. A Semantic Gateway Architecture for Systen Integration in Networked Manufacturing[J]. Key Engineering Materials, 2010, 419(2): 453-456.

[16] Lei Qi, Song Yu-Chuan, Li Xian-Wang. Heterogeneous system integration framework and key technologies with the support of semantic gateway[J].
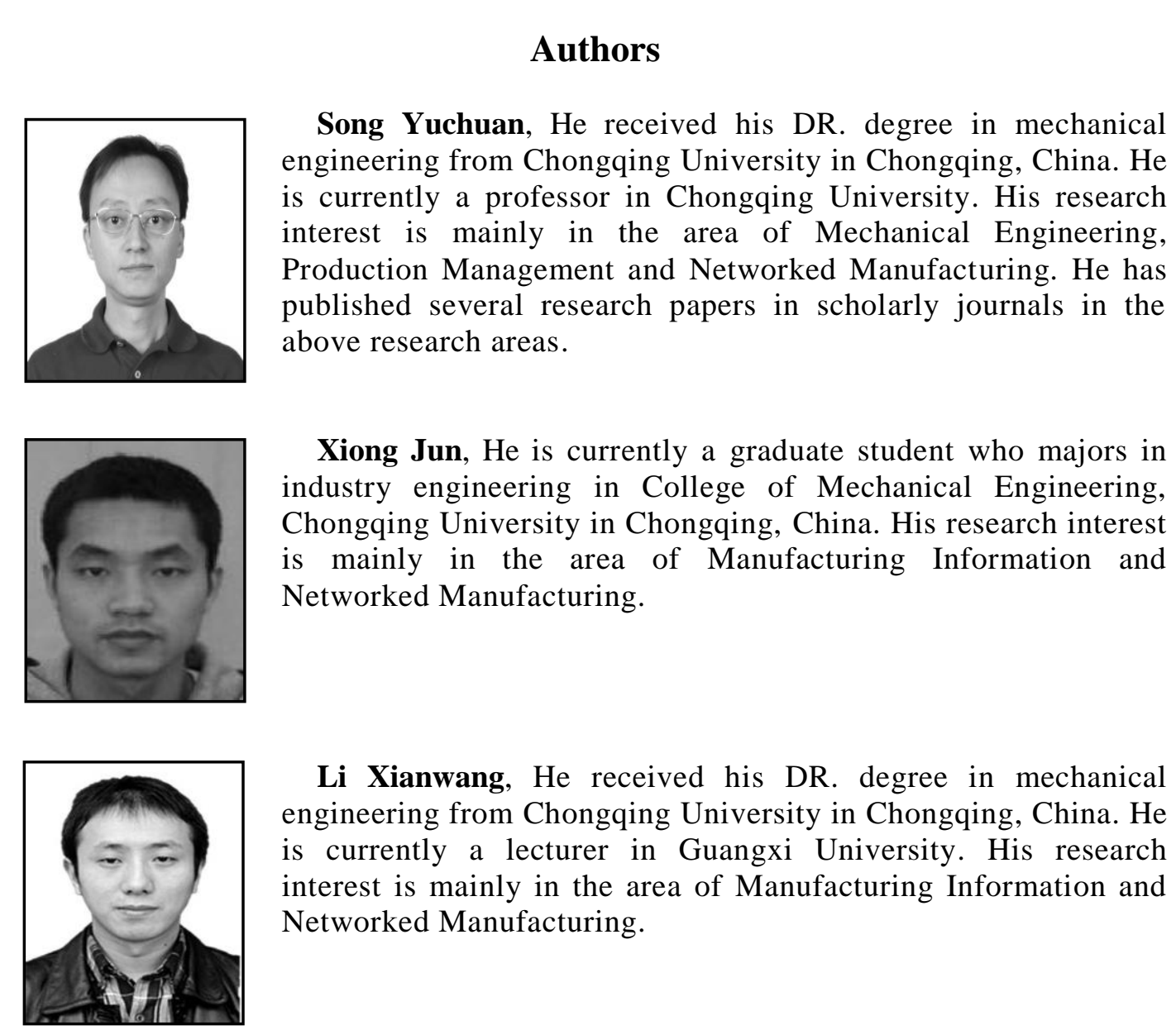

Li Xianwang, He received his DR. degree in mechanical engineering from Chongqing University in Chongqing, China. He is currently a lecturer in Guangxi University. His research interest is mainly in the area of Manufacturing Information and Networked Manufacturing. 


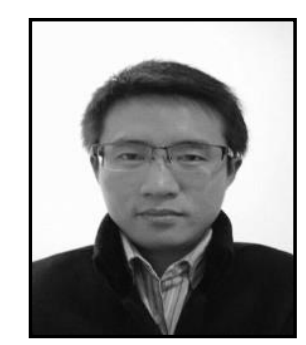

Chen Xuehai, He received his DR. degree in mechanical engineering from Chongqing University in Chongqing, China. He is currently a lecturer in Hubei University of Technology. His research interest is mainly in the area of Manufacturing Information and Networked Manufacturing.

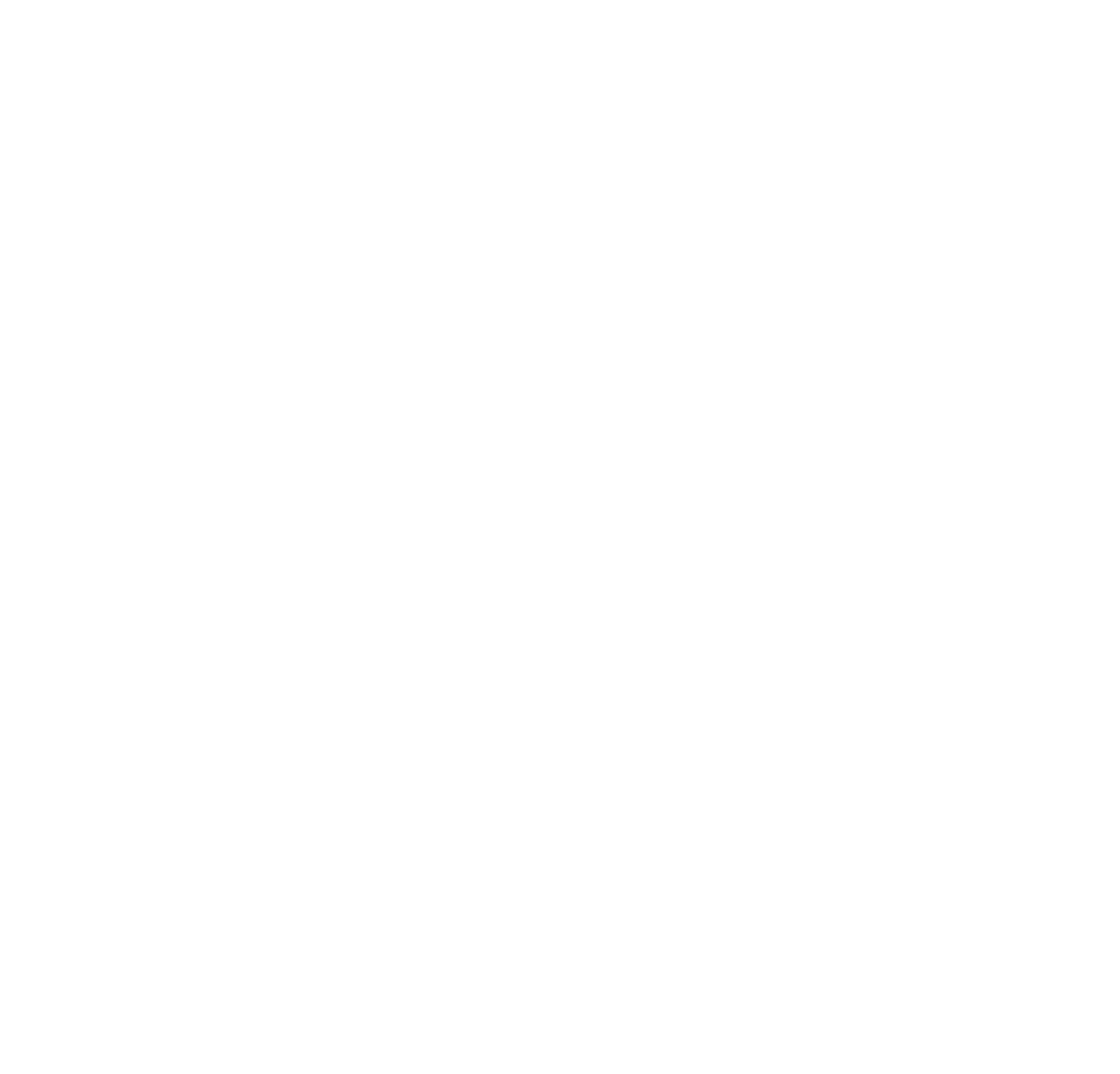

